\title{
Relación de los estilos de liderazgo, cohesión grupal, potencia de equipo y rendimiento en jugadores de fútbol no profesionales
}

\section{Relationship of Leadership Styles, Group Cohesion, Power Equipment and Performance in Non-Professional Soccer Players}

\author{
Elisa HuÉsCAR HernándeZ \\ Universidad Miguel Hernández de Elche, España \\ ORCID: http://orcid.org//0000-0002-2713-4506 \\ Clara lópez Mora \\ Universidad Miguel Hernández de Elche, España \\ Eduardo Cervelló Gimeno \\ Universidad Miguel Hernández de Elche, España
}

a Autor de correspondencia. Correo electrónico: elisahuescarhernandez@gmail.com

Para citar este artículo: Huéscar Hernández, E., López Mora, C., \& Cervelló Gimeno, E. (2017). Relación de los estilos de liderazgo, cohesión grupal, potencia de equipo y rendimiento en jugadores de fútbol no profesionales. Universitas Psychologica, 16(4), 1-14. ht tps://doi.org/10.11144/Javeriana.upsy16-4.relc

\section{RESUMEN}

El objetivo del presente trabajo fue analizar las relaciones entre tipos de liderazgo (tarea y social), cohesión, potencia de equipo y rendimiento en 334 futbolistas federados de las categorías Alevín, Cadete, Juvenil y Absoluta, con una edad media de 15.97 (DE = 3.31). Los cuestionarios utilizados fueron de Liderazgo auténtico (ALQ), tarea (LSS), Cohesión grupal (CG), Potencia de equipo (CPEA) y rendimiento, que mostraron índices de ajuste óptimos $\left(\chi^{2} / \mathrm{DF}=1.313 p=0\right.$; CFI $=0.997$; TLI $=$ 0.994; RMSEA $=0.032$; SRMS $=0.0343)$. Los resultados indican que el estilo de liderazgo, especialmente liderazgo tarea $(p=0.37)$ influye indirectamente en el rendimiento a través de la CG $(p=0.17)$ y CPE $(p$ $=0.21$ ). Como conclusión, se propone la mejora del rendimiento grupal a partir de la optimización de modelos de conducta individuales.

Palabras clave

liderazgo; cohesión grupal; potencia de equipo; rendimiento; fútbol.

\footnotetext{
ABSTRACT

The objective was to analyze the relationships between types of leadership (task and social), cohesion, team potency and performance in 334 federated players of the categories Alevín, Cadet, Juvenile and Absolute, with a mean age of $15.97(\mathrm{SD}=3.31)$. The questionnaires used were Authentic Leadership (ALQ), Task (LSS), Group Cohesion (CG), Team Power (CPEA), and Performance that showed optimal adjustment indexes $\left(\chi^{2} / \mathrm{DF}=1.313 p=0 ; \mathrm{CFI}=0.997 ; \mathrm{TLI}=0.994 ; \mathrm{RMSEA}=0.032 ;\right.$ SRMS $=0.0343)$. The results indicate that leadership style, especially leadership task $(p=0.37)$ indirectly influences performance through CG $(p=0.17)$ and CPE $(p=0.21)$. In conclusion, it is proposed to improve group performance by optimizing individual behavior models.

Keywords

leadership; group cohesion; power equipment; performance; football.
} 
La relevancia de las variables psicológicas en el ámbito del deporte competitivo para determinar el éxito es un hecho bien asumido por la comunidad científica actual (Abenza, Olmedilla, \& Ortega, 2010). Sin embargo, pese a que numerosos estudios vienen focalizando sobre la ansiedad, el estrés, la motivación, el ajuste emocional, la autorregulación o la cohesión (Adie, Duda, \& Ntoumais, 2012; Boardley, \& Kavussanu, 2010) como algunos de los factores sobre los que intervenir, un modelo que ayude a comprender no solo la relación entre estas variables (Leo, Sánchez-Miguel, SánchezOliva, Amado, \& García-Calvo, 2013; Martin, Carron, Eys, \& Loughead, 2012; Sánchez-Oliva, Leo, Sánchez-Miguel, Amado, \& García-Calvo, 2010), sino también su potencial contribución hacia la optimización de los resultados en los deportistas (Myers, Payment, \& Feltz, 2004), se hace necesario. La estrecha relación conceptual que en ocasiones mantienen estas variables hace aún más complejos los intentos de acotar un modelo válido subyacente a la estructura de los grupos eficaces. Permitir a técnicos, entrenadores y demás personas que están al frente de un equipo deportivo optimizar esfuerzos conociendo qué aspectos serían los más adecuados para conducir a los deportistas hacia un mayor rendimiento en función de su modelo de conducta, podría garantizar la continuidad en la práctica a largo plazo.

En este sentido, numerosos trabajos vienen planteando que la dirección de equipos basada en un liderazgo eficaz se presenta como la clave para mejorar el rendimiento deportivo (Bryman, 1992; Riemer, 2007). Entendemos por estilo de liderazgo al patrón de conducta estable que muestra la persona que tiene a su cargo la dirección de un equipo (Eagly, Johannesen-Schmidt, \& Van Engen, 2003). Sin embargo, el líder constituye, además, una parte fundamental de la estructura grupal, siendo determinante en la calidad de las relaciones sociales de sus integrantes (García-Calvo et al., 2014; Rewey, Dansereau, Dees, Skaggs, \& Pitre, 1992). Algunos estudios señalan que un líder con verdadera vocación prefiere tratar a sus seguidores de igual a igual, adaptando su conducta en función de los intereses del grupo y según el contexto para la consecución de las metas propuestas, pudiendo centrarse bien en la gestión eficaz de las tareas por realizar o bien en la gestión de la calidad de las relaciones sociales existentes en el grupo (Carron, Burke, \& Prapavesssis, 2004; Carron, Colman, Wheeler, \& Stevens, 2002).

Los estilos de liderazgo tarea y social han formado parte de muchos de los estudios clásicos sobre liderazgo (Molero, 1995), contemplándose como dos dimensiones independientes. Durante las décadas de los 70 y 80 , el liderazgo tarea fue subrayado en la investigación (Chelladurai, 1990, 1993); después, los aspectos referentes a la gestión socioemocional del grupo resurgieron como recurso fundamental para el líder eficaz mediante el liderazgo auténtico.

Así pues, un líder cuyo modelo de conducta está orientado a la tarea se define por dedicar su esfuerzo preferentemente a la ejecución del trabajo y a la consecución de los objetivos marcados. Este estilo de dirección de equipos es percibido por el grupo como una persona que está preparada, es experta y competente en esa labor y, en consecuencia, confían en la buena gestión de los procesos implicados para alcanzar las metas propuestas. En esta li\# nea, Chelladurai (1990; Chelladurai \& Saleh, 1980) creo\# el modelo multidimensional del liderazgo, especi\# fico para el ámbito del deporte. Este modelo entiende el liderazgo como un proceso interaccional y adaptativo, en el que la efectividad del li\#der, esto es, el rendimiento y la satisfacción de los deportistas, estaría influida por las caracteri\#sticas situacionales, las de los deportistas y las del propio entrenador. Derivado del modelo de Chelladurai, para evaluar estos aspectos, se creó la Escala de Liderazgo para el Deporte (LSS) (Chelladurai \& Saleh, 1978). Esta escala ha sido adaptada al deporte del fútbol recientemente en un trabajo aún en revisión por Ruiz-Barquín y De la Vega-Marcos, versionando dicho instrumento hacia la percepción de los entrenadores (LSS-3) (2015).

Respecto al concepto de líder preferentemente centrado en fomentar las relaciones 
interpersonales positivas, encontramos el concepto de liderazgo auténtico (Avolio \& Gardner, 2005; Luthans \& Avolio, 2003; Shamir \& Eilam, 2005; Walumbwa, Luthans, Avey, \& Oke, 2011). La "autenticidad" inherente a este concepto tiene que ver con la correspondencia entre los pensamientos y las acciones de la persona que dirige a un grupo. Esta congruencia está relacionada con los aspectos éticos y morales del liderazgo, y favorece la autorrealización tanto del líder como de las personas que trabajan con él (García-Guiu, Molero, Moya, \& Moriano, 2015; Moriano, Molero, \& Lévy-Mangin, 2011). Poner en valor las diferencias individuales, focalizar sobre los talentos frente a las debilidades y fomentar la autonomía y las relaciones del grupo, serían algunas de las claves sobre las que se basa este modelo de conducta. Sin embargo, para que este patrón se desarrolle con éxito, resulta fundamental que el líder, además de practicar estos valores anteriores con el grupo, sirva de referente social para las personas que lo componen, esto es, que sea percibido por el grupo como una persona que tiene interiorizado y pone en práctica los principios que les definen, no solo en el ámbito profesional, sino también, en el personal y social. En cuanto a la medida de este modelo, el Autentic Leadership Questionnaire (ALQ) es el cuestionario que Walumbwa, Avolio, Gardner, Wernsing y Peterson (2008) desarrollaron, aplicado al ámbito organizacional y que recientemente ha sido validado al español por Moriano et al. (2011). Según este instrumento, el liderazgo auténtico se divide en cuatro factores: Conciencia de sí mismo (autoconcepto del líder), Transparencia en las relaciones (sinceridad en la interacción), Procesamiento equilibrado (estilo de trabajo analítico) y Moral internalizada (autorregulación de la conducta acorde a los principios y valores personales frente a las presiones). Los estudios que se han llevado a cabo con este instrumento en diferentes países confirman la relación positiva de este estilo de conducta con la efectividad, el esfuerzo extra, la satisfacción (Moriano et al., 2011), el compromiso y el rendimiento laboral (Caza, Bagozzi, Woolley, Levy, \& Barker Caza, 2010; Clapp-Smith, Vogelgesang, \& Avey, 2009).
Sin embargo, tanto el ALQ en su versión original de Walumbwa et al. (2008) como la validación al español de Moriano et al. (2011) están basados en el ámbito organizacional o de la empresa, no encontrando hasta el momento estudios aplicados a entornos educativos y/o deportivos.

Pese a que una cierta flexibilidad en los comportamientos del líder en función de las necesidades o demandas del grupo (bien hacia los resultados, bien hacia los aspectos sociales) ha sido contemplada como posible por Bass (2004), aludiendo al liderazgo transformacional (Bass, 1990), incluso encontrándose altas correlaciones entre los dos estilos (Molero, Cuadrado, Navas, $\&$ Morales, 2007), un modelo que los incluya a ambos en una misma persona pudiendo ser utilizados de forma gradual en pro del rendimiento, no se ha contemplado hasta el momento en los estudios de investigación. Así pues, aunque desde la década de los noventa numerosos estudios han demostrado que ambos patrones de conducta de liderazgo, tarea y social, son eficaces en los grupos organizacionales (Bass, 1990; Molero et al., 2007), sin embargo, en los últimos años, en el ámbito de la actividad físico-deportiva, los estudios se vienen centrando sobre todo en el análisis de las relaciones sociales inherentes a los grupos deportivos, dejando de lado la gestión del grupo centrada en la tarea.

Junto a estos aspectos, por otro lado, la evidencia científica señala que el tipo de liderazgo percibido influye en el rendimiento, pero, además, otras variables de naturaleza social participan en el funcionamiento óptimo del equipo como son la cohesión grupal (García Calvo et al., 2014; García-Guiu et al., 2015; Leo, Garci\# a Calvo, Parejo, Sa\#nchez, \& Garci\#aMas, 2009; Paskevich, Estabtooks, Brawley, \& Carron, 2001) y la potencia de equipo (León, Mendo-Lázaro, Felipe-Castaño, Polo del Río, \& Fajardo-Bullón, 2017). Sin embargo, este último concepto no ha sido estudiado aún en el ámbito deportivo, como tampoco existen estudios que relacionen ambas variables con los estilos de liderazgo (tarea y social) y con el rendimiento.

Así pues, la cohesión de equipo es un proceso que se refleja en la tendencia de un grupo para permanecer unido en su propósito de conseguir 
o bien los objetivos instrumentales propuestos (Carron \& Chelladurai, 1981) o bien satisfacer sus necesidades afectivas (Calvo, Marcos, Miguel, Jiménez, \& Gimeno, 2008; Carron, Widmeyer, \& Brawley, 1985). Este último aspecto está relacionado con el componente social de la cohesión, que hace referencia a las relaciones de amistad y socialización de los miembros de un grupo, mientras que la consecución de objetivos instrumentales estaría relacionada con el componente tarea de la cohesión; explica la unión de las personas de un grupo para hacer frente a las actividades propuestas y mejorar el rendimiento colectivo, se trata de unir esfuerzos para alcanzar un objetivo común. Pese a la gran connotación social del término "cohesión", diferentes trabajos (Carron et al., 2002) señalan la importancia de la cohesión a la tarea para mejorar el rendimiento colectivo frente a la cohesión social. En este sentido, el reciente trabajo de Navarro-Patón, Mecías, Basanta y Lojo (2016) con jugadores de fútbol sala de tres equipos de máxima categoría, señala que los equipos mejor clasificados obtienen mejores puntuaciones en cohesión grupal.

Por otro lado, la potencia de equipo es un concepto definido por Guzzo, Yost, Campbell y Shea (1993) y se refiere a las creencias colectivas existentes en el grupo de que este puede ser efectivo. Se trata de una variable con origen motivacional muy importante, ya que su existencia podría influir en las actitudes de los integrantes de un equipo para realizar con éxito una tarea. Pese a que en el contexto deportivo no hemos encontrado trabajos que utilicen esta variable como predictora del rendimiento de los deportistas, los hallazgos del trabajo de León et al. (2017), con una muestra de estudiantes universitarios, apuntan a que este constructo se presenta como un factor esencial de la motivación grupal, pudiendo estar muy cercano a la cohesión y a la percepción del rendimiento o eficacia colectiva, dadas las connotaciones conductuales positivas que implica. De hecho, en el mencionado trabajo, el constructo potencia de equipo solamente aumentaba en el grupo en el que se utilizaban técnicas de naturaleza cooperativa. De esta forma, por su naturaleza social y su relación ya demostrada en otros contextos con el rendimiento grupal (Lester, Meglino, \& Korsgaard, 2002; Sivasubramaniam, Murry, Avolio, \& Jung, 2002), la potencia de equipo podría complementar la influencia positiva que constatan los estudios que la cohesión tarea ejerce respecto al rendimiento, al ser quizá un término más preciso y completo que la cohesión social para definir el sentimiento de unión entre las personas que se identifican con un grupo, sin perder de vista que estas relaciones son un medio para conseguir un fin, esto es, un objetivo común. Este último aspecto no lo incluye el factor social de la cohesión grupal, quedando meramente en una medida de los vínculos de amistad existentes entre los integrantes de un grupo.

La argumentación anterior justificaría la importancia de llevar a cabo un estudio que permita valorar de forma fiable la influencia de la cohesión grupal y la potencia de equipo sobre el rendimiento, a partir de la diferenciación de estilos de conducta de liderazgo enfocados o bien en la tarea o bien en las relaciones grupales.

Para verificar el orden causal planteado entre los factores mencionados arriba, sería muy importante determinar qué relaciones existen entre ambos tipos de liderazgo y el rendimiento, así como situar el papel de la cohesión y la potencia de equipo entre los diferentes patrones de conducta de liderazgo planteados y el rendimiento de los jugadores. Aunque existe literatura científica que relaciona algunos de los conceptos que se mencionan (Molero et al., 2007), de forma aislada no existe, sin embargo, ningún estudio que haya comprobado estas relaciones mediante un modelo unificador. Así pues, el principal objetivo de este trabajo es comprobar qué estilo de liderazgo (más basado en la gestión eficiente de las tareas o en la gestión eficiente de las relaciones sociales del grupo) tiene más peso en la mejora de la cohesión grupal, la potencia de equipo y, en última instancia, el rendimiento. Secundariamente, comprobar la robustez de algunas medidas de estas variables no empleadas antes en el ámbito deportivo, también será un objetivo del trabajo. 
La hipótesis de trabajo es que, dada la naturaleza del ámbito deportivo, el estilo de liderazgo basado en la gestión eficaz de las tareas será un predictor más potente de la cohesión grupal y la potencia de equipo que el liderazgo basado en la gestión social del grupo, y a su vez, se relacionará de forma más directa con el rendimiento. Igualmente, se espera que el aumento en la percepción de potencia de equipo, se relacionará positivamente con el rendimiento.

\section{Método}

\section{Diseño}

Para dar respuesta a los objetivos de investigación, se optó por un diseño no experimental, explicativo con variables observables, transeccional y descriptivo, bajo un enfoque cuantitativo, constituyéndose en una investigación de tipo exploratorio y descriptivo. La recolección de información se realizó a través de una fuente primaria, la batería de cuestionarios administrada a los deportistas, y una fuente secundaria, el informe de los entrenadores relativo al rendimiento observado de los deportistas.

\section{Participantes}

La muestra de este estudio se obtuvo a través de muestreo incidental. Está conformada por 334 futbolistas varones, federados pertenecientes a categorías Alevín, Cadete, Juvenil y Absoluta con una edad media de $15.97(\mathrm{DE}=3.31)$. Todos pertenecen a clubes de fútbol de las regiones de Murcia y Alicante (España). La muestra se distribuye de forma normal, situándose parte de los sujetos en niveles tanto bajos como altos en todos los factores. Esta observación se confirma a través del análisis no paramétrico de Kolmogorov-Smirnoff. En la Tabla 2 se pueden observar los resultados descriptivos.

\section{Definición de variables}

Las variables que se consideraron en este estudio fueron extraídas de los hallazgos de las investigaciones especializadas reseñadas en apartados anteriores. Además de las variables medidas a través de instrumentos estandarizados, se contempló la edad y categoría federada del deportista (Tabla 1).

\section{TABLA 1}

Definiciones de las variables a nivel conceptual de la investigación

\begin{tabular}{ll}
\hline \multicolumn{1}{c}{ Variable } & \multicolumn{1}{c}{ Definición conceptual } \\
\hline Liderazgo auténtico & $\begin{array}{l}\text { Patrón de conducta estable que muestra la persona que } \\
\text { tiene a su cargo la dirección de un equipo y se basa en } \\
\text { la correspondencia entre los pensamientos y las } \\
\text { acciones. } \\
\text { Patrón de conducta estable que muestra la persona que } \\
\text { tiene a su cargo la dirección de un equipo y se basa en } \\
\text { la ejecución del trabajo y a la consecución de los } \\
\text { objetivos marcados } \\
\text { Tendencia de un grupo para permanecer unido en su } \\
\text { propósito de conseguir o bien los objetivos } \\
\text { instrumentales propuestos o sus necesidades afectivas. } \\
\text { Cohesión Grupal }\end{array} \quad \begin{array}{l}\text { Conjunto de creencias colectivas existentes en el grupo } \\
\text { de que éste puede ser efectivo. }\end{array}$ \\
Valoración de la ejecución objetiva del grupo (ganar o \\
perder) o subjetiva del jugador y entrenador (balance \\
de una ejecución puntual en relación a la práctica \\
habitual).
\end{tabular}

Fuente: Adaptado de Moriano et al., (2011),

Crespo, Balaguer, y Atienza (1994), y

Marcos, Miguel, Oliva, y Calvo, (2010).

\section{Instrumentos}

Cuestionario de liderazgo auténtico (ALQ)

Se utilizó la adaptación española (Moriano et al., 2011) del Cuestionario de Liderazgo Auténtico (ALQ), originalmente desarrollado por Walumbwa et al. (2008). Lo componen un total de 12 ítems, con escala tipo Likert de 7 puntos y se divide en cuatro factores de primer orden y un factor de segundo orden denominado Liderazgo Auténtico. Los factores de primer orden son: Transparencia en las relaciones ("Creo que mi entrenador dice exactamente lo que piensa"), Moral internalizada ("Muestra comportamientos que son acordes a sus creencias"), Conciencia de sí mismo ("Solicita puntos de vista diferentes a sus opiniones") y Procesamiento equilibrado ("Busca la opinión de los demás para mejorar las relaciones con ellos"). Los resultados del análisis factorial confirmatorio efectuado, considerando 
el factor de segundo orden y los cuatro factores de primer orden, fueron: $\chi^{2}(50)=2.388, p=0$; CFI $=0.94 ; \mathrm{TLI}=0.921 ; \mathrm{RMSEA}=0.065 ; \mathrm{SRMS}$ $=0.056$.

Escala de Liderazgo en el Deporte (LSS)

Instrumento validado por Crespo, Balaguer y Atienza (1994), original de la versión española de Liderazgo para deportes de Chelladurai y Saleh (1980). Para este estudio, se emplean únicamente los ítems correspondientes a los factores de feedback positivo ("Mi entrenador felicita a un jugador delante de los demás por jugar bien") y Entrenamiento e instrucción ("Mi entrenador se preocupa de que los jugadores entrenen al máximo de sus capacidades"), ya que los complementarios (Conducta democrática, Conducta autocrática, Apoyo social) están referidos a otras dimensiones del liderazgo, y algunas de ellas ya se han recogido con el instrumento anterior. La versión original de esta escala está compuesta por 40 ítems, en este estudio se implementaron únicamente 17 . Las indicaciones previas fueron: "Creo que mi entrenador...”. Este instrumento se puntúa en una escala tipo Likert de 5 puntos. Los índices de ajuste de este cuestionario, considerando dos factores de primer orden y un factor de segundo orden, al que denominamos Liderazgo Tarea fueron: $\chi^{2} / \mathrm{DF}=2.002 p=0 ; \mathrm{CFI}=0.94 ; \mathrm{TLI}=$ $0.931 ; \operatorname{RMSEA}=0.056 ;$ SRMS $=0.0488$.

\section{Cohesión grupal (GEQ)}

Se utilizó el instrumento validado por Marcos, Miguel, Oliva y Calvo (2010) de la versión original de Carron et al. (1985). Consta de 12 ítems agrupados en cuatro factores de primer orden (tres ítems por factor) y un factor general (Cohesión grupal), de segundo orden. Los factores de primer orden son: Atracción individual social ("A los miembros del equipo les gustaría salir de fiesta juntos"), Atracción individual hacia la tarea ("Todos los jugadores asumen la responsabilidad ante un mal resultado del equipo"), Integración individual social ("Algunos de mis mejores amigos están en este equipo") e Integración individual tarea ("Me gusta el estilo de juego que tiene este equipo"). La sentencia previa fue: "Indica hasta qué punto las frases se corresponden con lo que piensas". Las respuestas se puntúan con una escala tipo Likert de 7 puntos. Los índices de ajuste de este cuestionario, considerando cuatro factores de primer orden y un factor de segundo orden, fueron: $\chi^{2} / \mathrm{DF}=3.513 p=0 ; \mathrm{CFI}=0.9 ; \mathrm{TLI}=$ $0.871 ; \mathrm{RMSEA}=0.078 ; \mathrm{SRMS}=0.0708$.

\section{Potencia de Equipo (CPE)}

La potencia de equipo se evalúa a través del Cuestionario de Potencia de Equipos de Aprendizaje (CPEA) diseñado y validado recientemente por León et al. (2017). Consta de ocho ítems en formato Likert con 10 intervalos en forma numérica del 1 (totalmente de acuerdo) al 10 (totalmente en desacuerdo) ("Mi equipo tiene confianza en sí mismo" o "Mi equipo tiene un alto grado de eficacia"). La sentencia previa fue: "A continuación, te presentamos una serie de cuestiones relacionadas con las creencias sobre si tu equipo puede desempeñarse de forma exitosa". Los índices de ajuste de este cuestionario son: $\chi^{2} /$ $\mathrm{DF}=3.363 p=0 ; \mathrm{CFI}=0.959$; TLI $=0.942$; RMSEA $=0.055 ;$ SRMS $=0.0377$.

\section{Rendimiento}

El rendimiento de los deportistas se evalúa a través de la puntuación media de dos dimensiones: rendimiento subjetivo interno y rendimiento subjetivo externo. Esta medida subjetiva ya ha sido utilizada en trabajos previos, en deportes colectivos e individuales, en los que el rendimiento objetivo del grupo (ganar o perder), no es una medida realista del nivel de ejecución individual (Cervelló, Santos-Rosa, García-Calvo, Jiménez, \& Iglesias, 2007).

El rendimiento subjetivo interno (percibido por el jugador de forma subjetiva o interna), se administró justo después de terminar la competición, mediante un solo ítem ("¿Cómo crees que ha sido tu rendimiento durante el partido, comparado con cómo rindes 
habitualmente?"). Este ítem se responde en una escala tipo Likert, con un rango de respuesta de 1 a 10 , donde 1 corresponde a mucho peor de lo habitual y 10 a mucho mejor de lo habitual.

El rendimiento subjetivo externo (percibido por el entrenador de forma externa) también se administró justo después de terminar la competición mediante un solo ítem ("iCómo crees que ha sido el rendimiento de este jugador durante el partido comparado con cómo rinde habitualmente?"), contestado por el entrenador, tras ver el número de camiseta que pertenece a cada jugador en la cuartilla de cuestionario. Este ítem se responde en una escala tipo Likert, con un rango de respuesta de 1 a 10 , donde 1 corresponde a mucho peor de lo habitual y 10 a mucho mejor de lo habitual. Ambas medidas correlacionaron significativamente $(r=0.44)$ y presentan un alpha aceptable al ser considerada simultáneamente $(0.71)$, por lo que se colapsaron en una única medida de rendimiento.

\section{Procedimiento}

Para llevar a cabo la recogida de datos, se ha creado un protocolo de actuación con el objetivo de unificar criterios y minimizar variables extrañas. En primer lugar, los investigadores se ponen en contacto con los clubes para solicitar su participación en el estudio y se solicita autorización de los tutores legales de los menores, indicándoles los objetivos de la investigación y el posterior tratamiento de datos y resultados (consentimiento informado), garantizando siempre su anonimato (World Medical Association, 2000). Los jugadores también han sido informados de los fines de la investigación. La batería de cuestionarios se administra a los jugadores en dos momentos distintos. Durante los entrenamientos, se administra la primera parte de la batería, y en un segundo momento postcompetición, se procede a medir la valoración del rendimiento a jugadores y entrenadores.
Análisis de datos

El tratamiento de datos en el presente trabajo se ha realizado en distintos niveles. Primeramente, a nivel de escalas se ha realizado un análisis descriptivo referido a normalidad de la muestra, media y desviación estándar. Además, se estimó la consistencia interna de las subescalas mediante el coeficiente alfa de Cronbach. Complementariamente, y como apreció en el apartado de Método, los datos han sido sometidos a análisis factorial confirmatorio (AFC) con el método de Estimación de Parámetros de Máxima Verosimilitud.

Para determinar la bondad de ajuste del path del modelo planteado en la hipótesis, se tuvieron en cuenta los diferentes índices de bondad del ajuste, los coeficientes estandarizados, el porcentaje de varianza explicado y la significación estadística. Siguiendo las directrices de Brown (2006) y Kline (2005), los índices de bondad de ajuste utilizados fueron los siguientes: el test chi-cuadrado, el índice de ajuste comparativo (CFI), el Tucker Lewis Index (TLI), el error cuadrático medio de aproximación (RMSEA) y la raíz del residuo estandarizado cuadrático medio (SRMR).

\section{Resultados}

Fiabilidad, descriptivos y correlaciones de todas las variables

En la Tabla 2, se pueden observar los estadísticos descriptivos y análisis de fiabilidad de las variables que forman parte del estudio (media, desviación estándar y alpha de Cronbach). Algunos de los factores (fundamentalmente los de primer orden) presentan alpha moderados, mejorando este valor cuando observamos los factores de segundo orden. Respecto a las correlaciones, se encuentra una relación moderada entre los factores del Liderazgo Tarea (Feedback positivo y Entrenamiento e instrucción) y el Liderazgo Auténtico (Transparencia en las relaciones, Moral Internalizada, Procesamiento equilibrado y Conciencia de sí mismo). Además, 
los factores de liderazgo Tarea correlacionan en mayor medida con los elementos de la cohesión grupal (IGT, IGS), mientras que las correlaciones presentadas por los factores de liderazgo auténtico, aunque también son significativas, se presentan como inferiores. Lo mismo ocurre con el factor Potencia de Equipo, las correlaciones presentadas por el liderazgo tarea son más elevadas que las presentadas al correlacionar con el liderazgo auténtico. Del mismo modo, es llamativo como la potencia de equipo correlaciona en mayor medida con las variables AIT y IGT, ambas orientadas a la tarea, lo cual vuelve a poner en relieve la importancia de la tarea en este marco de actuación. Cabe destacar las bajas correlaciones entre los dos tipos de liderazgo y el rendimiento, aunque en el caso del liderazgo tarea, esta sí que es significativa y positiva.

TABLA 2

Estadísticos descriptivos y correlaciones interna de las escalas

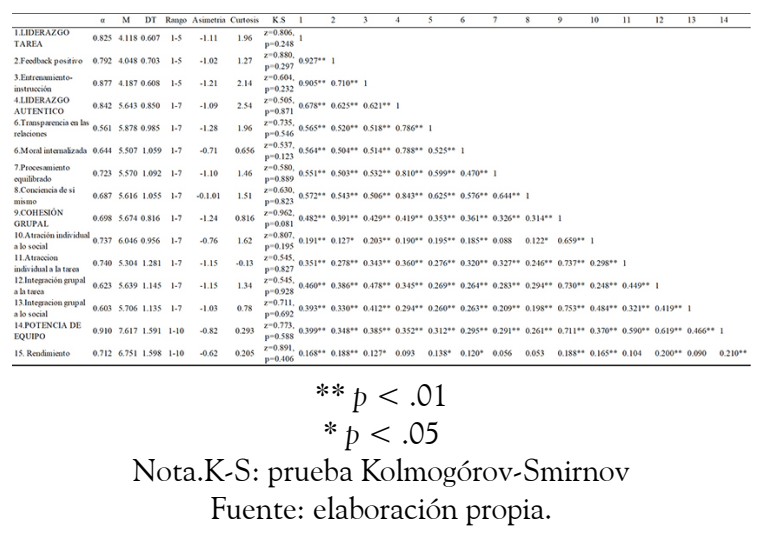

Análisis del modelo de relaciones entre variables (Path-análisis)

Se sometió a verificación un modelo de Path-análisis, que incluye factores sociales desencadenantes (liderazgo tarea y auténtico), consecuencias de primer orden (cohesión grupal y potencia de equipo) y consecuentes de segundo orden (rendimiento). La base estuvo constituida por los cinco constructos de segundo orden, dado que eran los que presentaban mejores niveles de fiabilidad. Para todas las variables, se utilizaron las medias entre factores. Se llevó a cabo el método de estimación de máxima verosimilitud.

Los índices de ajuste de este modelo fueron adecuados: $\chi^{2} / \mathrm{DF}=1.313 p=0 ; \mathrm{CFI}=0.997$; TLI $=0.994 ;$ RMSEA $=0.032 ;$ SRMS $=$ 0.0343 , mostrando excelentes niveles de ajuste. Los parámetros estandarizados se muestran en la Figura 1, donde se observa que ambos tipos de liderazgo predicen positivamente la cohesión, aunque el liderazgo tarea lo hace en mayor medida. Por su parte, la cohesión grupal predijo de forma positiva y significativa la potencia de equipo percibida por los jugadores. A su vez, dicha potencia predijo positivamente la percepción de rendimiento obtenido. Todos los parámetros fueron significativos.

\section{Figura 1.}

Path-analysis entre los estilos de liderazgo, cohesión grupal, potencia de equipo y rendimiento.

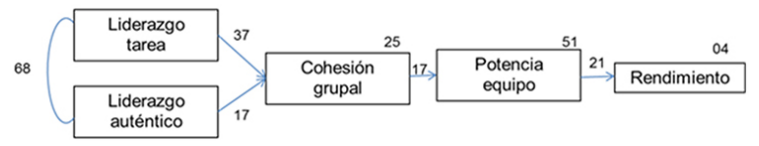

Fuente: elaboración propia.

\section{Discusión}

El propósito de este trabajo fue testar un modelo teórico que ayude a comprender cómo diferentes estilos de liderazgo (más basados en la gestión de las tareas del grupo, o más basados en la gestión del clima social del mismo) pueden afectar a variables como la cohesión, la potencia de equipo y el rendimiento. Desde un punto de vista general, los resultados del Path-análisis propuesto apoyaron las hipótesis planteadas, de forma parcial, mostrando predicción de ambos tipos de liderazgo sobre la cohesión de equipo, la cual predijo también la potencia de quipo. Esta última predijo el rendimiento, aunque de forma muy poco contundente. De esta forma, el modelo analizado permite un acercamiento a una explicación sobre la contribución de ambos estilos en la predicción del rendimiento deportivo.

Una visión más detallada de los resultados muestra que existen relaciones positivas entre 
la mayor parte de las variables estudiadas y los estilos de liderazgo (tarea y auténtico) analizados en el presente trabajo, reforzando así la idea, ya expresada en otros estudios (Avolio, Bass, \& Zhu, 2004), de que un líder puede manejar a la vez aspectos relacionados con los resultados o recompensas y con los aspectos transformacionales, implicados en el liderazgo auténtico. Así pues, sería esperable que la percepción por parte de los subordinados de que el líder gestiona eficazmente las recompensas en función de los resultados, esté contribuyendo -mediante el aumento de confianza que genera este hecho- a aumentar los aspectos sociales de identificación con el líder (Shamir, 1995). Concretamente, la relación entre estas variables ha sido analizada en algunos trabajos (Avolio et al., 2004; Molero et al., 2007), corroborando la estrecha relación entre los patrones de conducta relacionados con el liderazgo tarea y auténtico.

Particularmente, los resultados de este estudio revelaron que tanto el liderazgo tarea como el liderazgo auténtico se relacionaban positivamente con la cohesión grupal, mostrando, sin embargo, puntuaciones más altas las variables contempladas en el liderazgo tarea. Específicamente, sus dos dimensiones, esto es, Feedback positivo y Entrenamiento e instrucción, mostraron las mayores relaciones positivas significativas con las variables de la cohesión, resaltando la cohesión hacia la tarea. Este resultado está en la línea de los trabajos de Leo et al. (2013) o Myers et al. (2004) y Carron y Eys (2012), que subrayan que los estilos que fomentan las expectativas positivas creadas en las personas o la mejora de los resultados, ambos aspectos recogidos en el liderazgo tarea, favorecen conductas adaptativas en el grupo tales como la cohesión, lo que se traduciría en el aumento del compromiso grupal hacia la consecución de objetivos comunes. En este sentido, Chelladurai (1989) también apunta a la satisfacción como uno de los consecuentes del liderazgo tarea. Efectivamente, es probable que la satisfacción que reporta el hecho de reconocer tanto el esfuerzo de los jugadores mediante el Feedback positivo (FB) como también el compromiso percibido por parte del jugador de que el entrenador se interesa por maximizar las potencialidades de las personas que integran el grupo (EI), esté facilitando la adaptación intragrupal de sus miembros en busca de una determinada identidad como grupo. De esta forma, esta homogeneidad grupal de carácter social resultante, aunque termina reforzando los lazos sociales entre el grupo, como demuestran las correlaciones positivas entre el liderazgo auténtico y la cohesión, y como vienen confirmando estudios previos con variables similares como el compromiso o la satisfacción (Moriano, Palací, y Morales, 2007), parece no obstante, estar determinada por otros aspectos relacionados con la gestión eficaz de tareas, tales como la identificación de las personas con la dirección adecuada de los objetivos o el convencimiento del correcto desempeño de los roles individuales.

Respecto a la potencia de equipo, esta variable mostró relaciones positivas y significativas tanto con ambos estilos de liderazgo como con la cohesión de equipo, resultando de nuevo, tener su aporte más significativo con las dimensiones tarea de la cohesión. Hasta la fecha, los trabajos encontrados que incluyen la potencia de equipo se han limitado al ámbito educativo (León et al., 2017), siendo inexistentes los estudios científicos en el campo de la actividad física o el deporte. El concepto de potencia de equipo, aunque interrelacionado con la cohesión por su naturaleza social, está más centrado en la creencia colectiva de eficacia (Bandura, 1997). La cohesión, en cambio, implica la capacidad que muestra cada jugador de adaptarse al resto. En este sentido, es posible, tal y como muestra la predictibilidad de la potencia respecto a la cohesión de equipo, que solamente una vez cohesionados, esto es, cuando los jugadores se sienten adaptados y coherentes mutuamente, sean capaces de percibir que el grupo puede llegar lejos. Efectivamente, el trabajo de León et al. (2017), basándose en estudios anteriores (Johnson, Skon, \& Johnson, 1980), ya señala los beneficios de los escenarios cooperativos para los resultados tanto académicos, esto es, centrados en el resultado, (tarea) como sociales. 
Respecto al rendimiento, las variables analizadas no se relacionaron de forma consistente con el mismo. Revisando los estilos, únicamente el liderazgo tarea se relacionó con el rendimiento, si bien el valor de la correlación, aunque significativo, es bajo. Pese a que, como ya se ha indicado, en los últimos años la investigación parece centrarse en el liderazgo auténtico para favorecer los resultados en el grupo (Avolio \& Gardner, 2005), siendo inexistentes los trabajos que recojan el aporte fundamental de la gestión de tareas por parte del líder (Chelladurai, 1989) en pro de los resultados, los hallazgos de las correlaciones deberían ser incentivo para seguir explorando en esta línea.

Además, la visión del path muestra que ambos tipos de estilos de dirección de equipos parecen tener impacto sobre los resultados, a través de su efecto en otras variables grupales como la cohesión y la potencia de equipo. En esta línea, la posibilidad de una graduación en los estilos de liderazgo en lugar del manejo exclusivo de uno u otro, viene siendo señalada en la literatura científica sobre el tema, bajo el concepto de liderazgo transformacional (Bass, 1990; Molero, 1995), el cual contempla, en pro de los beneficios del equipo, la posibilidad de incluir bajo un mismo estilo de dirección tanto aspectos de gestión eficaz de tareas como de identificación personal respecto a actitudes, creencias y valores con los miembros del grupo.

Respecto a los resultados sobre la relación mediadora de la cohesión y la potencia de equipo con el rendimiento, León et al. (2017), refiriéndose al trabajo de naturaleza cooperativa, apuntan que cuanto más se consoliden en un grupo los recursos de interacción social mayor rendimiento y eficacia encontramos. Este aspecto estaría en consonancia con las altas puntuaciones halladas en el presente trabajo en relación con el factor cohesión. Así pues, determinadas características sociales pueden estar influyendo en los resultados positivos para con el grupo (Rewey et al., 1992; León, 2006). También Navarro, Quijano, Berger y Meneses (2011) señalan como indicadores de la eficacia de trabajo de un equipo a la potencia, la madurez grupal y la identificación de los miembros con el equipo, aspecto este último incluido en la cohesión grupal.

En definitiva, el presente modelo sugiere que, aunque ambos estilos de liderazgo tienen un impacto sobre la gestión del grupo en deporte, no se deberían olvidar los aspectos procedimentales aportados por el liderazgo tarea, ya que arrojan los resultados más destacados para el rendimiento, aunque se debe explorar de forma más profunda la solidez de estas relaciones. Además, parece que en este proceso garantizar la autopercepción de integración en el grupo del deportista que aporta la cohesión grupal, unido al sentimiento de valía y competencia que añade la potencia de equipo, situarían esta investigación más cerca de aspirar a la mejora de los resultados conjuntos. No obstante, se señalan algunas limitaciones que han de ser tenidas en cuenta cuando se interpreten estos resultados. En primer lugar, la medida de las variables mediante cuestionarios puede crear un error de varianza en el modelo. Además, los aspectos motivacionales implicados tanto en la cohesión como en la potencia de equipo hacen de este un modelo de los muchos otros que pueden existir, pudiendo estar el rendimiento afectado también por otras variables que aquí no se han contemplado.

También se debería plantear para futuros trabajos la medición del rendimiento deportivo con otro tipo de medidas que no sean tan generalistas, sino que se acote un poco más la naturaleza del rendimiento (técnico, táctico, físico, psicológico), de forma que se tenga una imagen más clara de cómo las variables grupales afectan al rendimiento y se adquiera un conocimiento más profundo del efecto del manejo de las variables colectivas en el rendimiento individual.

\section{Referencias}

Abenza, L., Olmedilla, A., \& Ortega, E. (2010). Efectos de las lesiones sobre las variables psicolo\#gicas en futbolistas juveniles. Revista Latinoamericana de Psicologi\#a, $42(2), 265-277$. 
Adie, J. W., Duda, J. L., \& Ntoumanis, N. (2012). Perceived coach-autonomy support, basic need satisfaction and the well-and illbeing of elite youth soccer players: A longitudinal investigation. Psychology of Sport and Exercise, 13, 51-59. https:// doi.org/10.1016/j.psychsport.2011.07.008

Avolio, B. J., Bass, B. M., \& Zhu, F. W. W. (2004). Multifactor leadership questionnaire: Manual and sampler set. Menlo Park ${ }^{\wedge}$ eCA CA: Mind Garden.

Avolio, B. J., \& Gardner, W. L. (2005). Authentic leadership development: Getting to the root of positive forms of leadership. The Leadership Quarterly, 16(3), 315-338. https://doi.org/10.1016/ j.leaqua.2005.03.001

Bandura, A. (1997). Self-efficacy and health behaviour. En A. Baum, S. Newman, J. Wienman, R. West \& C. McManus (Eds.), Cambridge handbook of psychology, health and medicine (pp. 160-162). Cambridge: Cambridge University Press.

Bass, B. M. (1990). Bass and Stogd ill's handbook of leadership. Nueva York: Free Press.

Bass, F. M. (2004). Comments on "A new product growth for model consumer durables the Bass model". Management Science, 50(Supl. 12), 1833-1840. https://doi.org/10.1287/ mnsc. 1040.0300

Boardley, I. D., \& Kavussanu, M. (2010). Efectos de la orientación hacia el objetivo y el valor percibido de la dureza en el comportamiento antisocial en el fútbol: el papel mediador de la desvinculación moral. Diario del Deporte y el Ejercicio de Psicología, $32(2), 176-192$.

Brown, A. T. (2006). Confirmatory factor analysis. Nueva York: Guilford Press.

Bryman, A. (1992). El carisma y el liderazgo en las organizaciones. Londres: Sage.

Calvo, T., Marcos, F. M., Miguel, P. A., Jiménez, R., \& Gimeno, E. C. (2008). Importancia de los aspectos motivacionales sobre el grado de cohesión en equipos de fútbol. Revista Iberoamericana de Psicología del Ejercicio y el Deporte, 3(1), 61-74.
Carron, A. V., Burke, S. M., \& Prapavessis, H. (2004). Self-presentation and group influence. Journal of Applied Sport Psychology, 16(1), 41-58. https:// doi.org/10.1080/10413200490260044

Carron, A. V., \& Chelladurai, P. (1981). Cohesion as a factor in sport performance. International Review of Sport Sociology, 16, 2-41.

Carron, A. V., Colman, M. M., Wheeler, J., \& Stevens, D. (2002). Cohesion and performance in sport: A meta analysis. Journal of Sport E8 Exercise Psychology, 24, 168-188.

Carron, A. V., \& Eys, M. A. (2012). Group Dynamics in Sport (4.a ed.). Morgantown, WV: Fitness Information Technology.

Carron, A. V., Widmeyer, W. N., \& Brawley, L. R. (1985). The development of an instrument to assess cohesion in sport teams: The Group Environment Questionnaire. Journal of Sport Psychology, 7(3), 244-266. https:// doi.org/10.1123/jsp.7.3.244

Caza, A., Bagozzi, R. P., Woolley, L., Levy, L., \& Barker Caza, B. (2010). Psychological capital and authentic leadership: Measurement, gender, and cultural extension. Asia-Pacific Journal of Business Administration, 2(1), 53-70. https:// doi.org/10.1108/17574321011028972

Cervelló, E., Santos-Rosa, F. J., Garci\#a Calvo, T., Jiménez, R., \& Iglesias, D. (2007). Young tennis players' competitive task involvement and performance: The role of goal orientations, contextual motivational climate, and coach-initiated motivational climate. Journal of Applied Sport Psychology, 19(3), 304-321. https:// doi.org/10.1080/10413200701329134

Chelladurai, P. (1989). Manual for the Leadership Scale for Sports. Manuscrito inédito, University of Western Ontario, Canadá.

Chelladurai, P. (1990). Leadership in sport: A review. International Journal of Sport Psychology, 21 (4), 328-354.

Chelladurai, P. (1993). Leadership. En R. N. Singer, M. Murphey \& L. K. Tennant (Eds.), Handbook of research on sport 
Elisa Huéscar Hernández, Clara lópez Mora, Eduardo Cervelló Gimeno.

psychology (pp. 647-671). Nueva York: Macmillan.

Chelladurai, P., \& Saleh, S. D. (1978). Preferred leadership in sports. Canadian Journal of Applied Sport Sciences, 3(2), 85-92.

Chelladurai, P., \& Saleh, S. D. (1980). Dimensions of leader behavior in sport: Development of a leadership scale. Journal of Sport Psychology, 2(1), 34-45.

Clapp-Smith, R., Vogelgesang, G. R., \& Avey, J. B. (2009). Authentic leadership and positive psychological capital the mediating role of trust at the group level of analysis. Journal of Leadership \& Organizational Studies, 15(3), 227-240. https:// doi.org/10.1177/1548051808326596

Crespo, M., Balaguer, I., \& Atienza, F. (1994). Análisis psicométrico de la versión española de la escala de liderazgo en el deporte de Chelladurai y Saleh en la versión entrenadores. Revista de Psicología Social Aplicada, 4(1), 5-28.

Eagly, A. H., Johannesen-Schmidt, M. C., \& Van Engen, M. L. (2003). Transformational, transactional, and laissez-faire leadership styles: A metaanalysis comparing women and men. Psychological Bulletin, 129, 569-591. https:// doi.org/10.1037/0033-2909.129.4.569

García-Calvo, T., Leo, F., Gonzalez-Ponce, I., Sánchez-Miguel, P. A., Mouratidis, A., \& Ntoumanis, N. (2014). Perceived coachcreated and peer-created motivational climates and their associations with team cohesion and athlete satisfaction: Evidence from a longitudinal study. Journal of Sports Sciences, 32(18), 1738-1750. https:// doi.org/10.1080/02640414.2014.918641

García-Guiu, C., Molero, F., Moya, M., \& Molero, J. A. (2015). Authentic leadership, group cohesion and group identification in security and emergency teams. Psicothema, 27(1), 59-64. https:// doi.org/10.1080/02134748.2014.987539

Guzzo, R. A., Yost, P. R., Campbell, R. J., \& Shea, G. P. (1993). Potency in groups: Articulating a construct. British Journal of Social Psychology,
32(1), 87-106. https://doi.org/10.1111/ j.2044-8309.1993.tb00987.x

Johnson, D. W., Skon, L., \& Johnson, R. (1980). Effects of cooperative, competitive, and individualistic conditions on children's problemsolving performance. American Educational Research Journal, 17, 83-94. https:// doi.org/10.3102/00028312017001083

Kline, R. B. (2005). Principles and practice of structural equation modeling (2.a ed.). Nueva York: The Guilford Press.

Leo, F. M., Garci\#a Calvo, T., Parejo, I., Sa\#nchez, P. A., \& Garci\#a-Mas, A. (2009). Aplicacio\#n de un programa de intervencio\#n para la mejora de la cohesio\#n y la eficacia en jugadores de baloncesto. Cuadernos de Psicologi\# a del Deporte, 9(1), 73-84.

Leo, F. M., Sánchez-Miguel, P. A., SánchezOliva, D., Amado, D., \& García-Calvo, T. (2013). El liderazgo y el clima motivacional del entrenador como antecedentes de la cohesión y el rol percibido en futbolistas semiprofesionales. Revista de Psicología del Deporte, 22(2), 361-370.

León, B., Mendo-Lázaro, S., Felipe-Castaño, E., Polo del Río, M. I., \& Fajardo-Bullón, F. (2017). Potencia de equipo y aprendizaje cooperativo en el ámbito universitario. Revista de Psicodidáctica, 22(1), 9-15. https:// doi.org/10.1387/RevPsicodidact.14213

León, B. (2006). Mediatory elements in the efficiency of cooperative learning: Previous training in social skills and group dynamics. Anales de Psicología, 22 (1), 105-112.

Lester, S. W., Meglino, B. M., \& Korsgaard, M. A. (2002). The antecedents and consequences of group potency: A longitudinal investigation of newly formed work groups. Academy of Management Journal, 45(2), 352-368. https://doi.org/10.2307/3069351

Luthans, F., \& Avolio, B. (2003). Authentic leadership development. En K. S. Cameron, J. E. Dutton \& R. E. Quinn (Eds.), Positive organizational scholarship: Foundations of a 
new discipline (pp. 241-258). San Francisco: Berrett-Koehler Publishers.

Marcos, F. M. L., Miguel, P. A. S., Oliva, D. S., $\&$ Calvo, T. G. (2010). Interactive effects of team cohesion on perceived efficacy in semi-professional sport. Journal of Sports Science $\mathbb{E}$ Medicine, 9(2), 320-325.

Martin, L. J., Carron, A. V., Eys, M. A., \& Loughead, T. M. (2012). Development of a cohesion inventory for children's sport teams. Group Dynamics: Theory, Research, and Practice, 16(1), 68-79. https:// doi.org/10.1037/a0024691

Molero, F. (1995). El estudio del carisma y del liderazgo carisma\#tico en las ciencias sociales: una aproximacio\#n desde la psicologi\# a social. Revista de Psicologi\#a Social, 10(1), 43-60.

Molero, F., Cuadrado, I., Navas, M., \& Morales, J. (2007). Relations and effects of transformational leadership: A comparative analysis with traditional leadership styles. The Spanish Journal of Psychology, 10(2), 358-368.

Moriano, J. A., Molero, F., \& Lévy-Mangin, J. P. (2011). Liderazgo auténtico. Concepto y validación del cuestionario en España. Psicothema, 23(2), 336-341.

Moriano, J. A., Palací, F. J., \& Morales, J. F. (2007). The Psychosocial Profile of the University Entrepreneur. Psychology in Spain, 11, 72-84.

Myers, N. D., Payment, C. A., \& Feltz, D. L. (2004). Relaciones recíprocas entre la eficacia colectiva y rendimiento del equipo en hockey sobre hielo femenino. Dinámica de Grupos: Teoría, Investigación y Práctica, 8(3), 182-195.

Navarro, J., Quijano, S. D. de, Berger, R., \& Meneses, R. (2011). Grupos en las organizaciones: herramientas ba\#sicas para gestionar la incertidumbre y ambigu\# edad crecientes. Papeles del Psico\#logo, 32, 17-28.

Navarro-Patón, R., Mecías, M., Basanta, S., \& Lojo, C. (2016). Análisis de la cohesión grupal de los equipos de fútbol sala de máxima categoría en Galicia (España).
Revista Iberoamericana de Psicología del Ejercicio y el Deporte, 11(2), 247-251.

Paskevich, D. M., Estabrooks, P. A., Brawley, L. R., \& Carron, A. V. (2001). Group cohesion in sport and exercise. Handbook of Sport Psychology, 2, 472-494.

Rewey, K. L., Dansereau, D. F., Dees, S. M., Skaggs, L. P., \& Pitre, U. (1992). Scripted cooperation and knowledge map supplements: Effects on the recall of biological and statistical information. The Journal of Experimental Education, 60(2), 93-107. https:// doi.org/10.1080/00220973.1991.10806582

Riemer, H. A. (2007). Multidimensional model of coach leadership. En S. Jowett \& D. Lavallee (Eds.), Social psychology in sport (pp. 57-73). Champaign, IL: Human Kinetics.

Ruiz-Barquín, R., \& de la Vega-Marcos, R. (2015). Adaptación de la escala de liderazgo LSS-3 al fútbol. Revista Internacional de Medicina y Ciencias de la Actividad Física y el Deporte, 15(60), 677-700. https://doi.org/10.15366/ rimcafd2015.60.005

Sánchez-Oliva, D., Leo Marcos, F. M., SánchezMiguel, P. A., Amado Alonso, D., \& García-Calvo, T. (2010). Relationship between motivational climate created by coach regarding self-determined motivation and the involvement through the practice. Revista Internacional de Ciencias del Deporte, 6, 177-195. https:// doi.org/10.5332/ricyde2010.02001

Shamir, B. (1995). Social distance and charisma: Theoretical notes and an exploratory study. Leadership Quarterly, 6, 19-47. https:// doi.org/10.1016/j.leaquia.2005.03.005

Shamir, B., \& Eilam, G. (2005). What's your story? A life-stories approach to authentic leadership development. The Leadership Quarterly, 16(3), 395-417.

Sivasubramaniam, N., Murry, W. D., Avolio, B. J., \& Jung, D. I. (2002). A longitudinal model of the effects of team leadership and group potency on group performance. Group and Organization 
Management, 27, 66-96. https:// doi.org/10.1177/1059601102027001005

Walumbwa, F. O., Avolio, B. J., Gardner, W. L., Wernsing, T. S., \& Peterson, S. J. (2008). Authentic leadership: Development and validation of a theory-based measure. Journal of Management, 34(1), 89-126. https:// doi.org/10.1177/0149206307308913

Walumbwa, F. O., Luthans, F., Avey, J. B., \& Oke, A. (2011). Authentically leading groups: The mediating role of collective psychological capital and trust. Journal of Organizational Behavior, 32(1), 4-24. https://doi.org/10.1002/job.653

World Medical Association. (2000). World Medical Association Declaration of Helsinki. Ethical principles for medical research involving human subjects. Bulletin of World Medical Association, 79(4), 373-374.

\section{Notas}

* Artículo de investigación. 